\title{
超强纳米颗粒溶液粘合剂
}

\author{
刘明倩方茜子王树涛* \\ (中国科学院理化技术研究所 中国科学院仿生材料与界面科学重点实验室 中国科学院大学 北京 100190)
}

\begin{abstract}
摘要 无论是自然界普遍存在的非共价键相互作用, 还是共价键结合, 都可以产生界面粘附. 粘附材料的应用需求广 泛, 涉及可穿戴设备、电子器件、医疗手术等诸多领域. 近些年来, 仿生结构粘附材料和仿生粘合剂已经得到了快速的 发展, 一些人工制备的粘附材料已投入实际应用. 然而, 结构微加工的局限性和合成路线的复杂性限制了它们的大规 模生产. 相比而言，纳米颗粒溶液提供了一种更简单和更容易实现强粘附的替代方法. 本文就最近报道的 “强各向异性 粘附的纤维素纳米晶体悬浮液” 作一亮点评述.
\end{abstract}

关键词 粘合剂; 纳米晶体; 自组装; 各向异性粘附

\section{Super Adhesive of Nanoparticle Solutions}

\section{Liu, Mingqian Wan, Xizi Wang, Shutao*}

(CAS Key Laboratory of Bio-inspired Materials and Interfacial Science, Technical Institute of Physics and Chemistry, Chinese Academy of Sciences, University of Chinese Academy of Sciences, Beijing 100190)

\begin{abstract}
Interfacial adhesion occurs by covalent bonding or noncovalent interactions that are ubiquitous in nature. Adhesive materials are widely desired in many fields including wearable devices, electronic devices, medical surgeries, etc. In recent years, bio-inspired structural adhesive materials and adhesives have been rapidly developed, and some artificial adhesive materials have been put into practical use. However, the restrictions of structural microfabrication and the complexity of synthetic routes limit their large-scale production. In contrast, the nanoparticle solutions provide a simpler and easier alternative to achieve strong adhesion. Here this article makes a highlight on the recent research of "strong anisotropic adhesion of cellulose nanocrystals suspension".
\end{abstract}

Keywords adhesive; nanocrystal; self-assembly; anisotropic adhesion

因为诸多领域的应用需求, 发展新型的高强度粘附 材料或胶粘剂一直倍受关注. 受自然界生物独特粘附现 象的启发, 科学家制备了仿壁虎的分级微纳米刚毛结 构 ${ }^{[1]}$ 、仿树蛙脚趾的表面微型通道 ${ }^{[2]}$ 和仿章鱼吸盘的粘 性贴片 ${ }^{[3]}$. 还有一些生物靠自身分泌的粘液实现粘附, 例如贻贝通过分泌足丝蛋白实现对基底的永久粘附，科 学家将足丝蛋白中的关键粘附基团邻苯二酚修饰到高 分子聚合物中, 合成人工粘接剂 ${ }^{[4]}$; 沙塔蠕虫依靠分泌 的粘附蛋白实现水下粘附, 受此启发, Waite 等 ${ }^{[5]}$ 设计了 一种由聚合物电解质复合物和有机溶剂组成的多功能 微孔粘合剂; 蜘蛛可以在干燥的屋檐下亦或是潮湿的湖 边利用精巧的蛛网进行捕食, 基于此, Lewis 等 ${ }^{[6]}$ 利用重 组蛛丝蛋白制备了强力粘合剂. 然而, 这些粘附材料的 制备需要繁杂的工艺流程或者多步的化学合成, 因此, 科学家们发展了一种新型粘附材料——纳米颗粒溶液 粘合剂.

2014 年, Leibler 课题组 ${ }^{[7]}$ 制备了一种含有二氧化硅 纳米颗粒溶液的粘合剂, 将该溶液滴在两个凝胶片之
间，纳米颗粒可以在两个凝胶之间起粘接作用(图 1a). 这种简易制备的纳米颗粒溶液可实现快速且高效的凝 胶或组织粘附. 在此基础上, Leibler 课题组 ${ }^{[8]}$ 进一步证 明了二氧化硅/氧化铁纳米颗粒水溶液可用于器官止血 和体内医疗器件粘结, 其中纳米颗粒起到了桥接缝合组 织伤口的作用(图 1b).

近日, 由 Tardy、Ejima 和 Rojas 等[9]组成的国际化 团队，制备了一种绿色的强粘合剂，仅通过来源于植物 的纤维素钠晶体，便可实现在主平面方向上的超强粘附 和垂直方向上的弱粘附. 该粘合剂具有潜在的应用前 景, 例如阻止易碎且高价值的机器组件或建筑元素的断 裂和提高可重复使用性.

该胶水的制备过程简单，如图 2a 所示，由纤维素纳 米晶体组成的水分散液在受限的蒸发作用下诱导纳米 晶体自组装, 在固体界面形成超结构的粘附层. 由于自 组装具有方向性，最终可形成高度有序的层次结构，导 致粘合剂与基底间显著的各向异性粘附强度. 在平面内 的剪切强度可以达到 $7 \mathrm{MPa}$ ，而垂直于平面的粘附强度

\footnotetext{
* E-mail: stwang@mail.ipc.ac.cn

Received April 18, 2020; published May 18, 2020.

Project supported by the National Natural Science Foundation of China (No. 21972155) and National Program for Special Support of Eminent Professional.

项目受国家自然科学基金(No. 21972155)和国家高层次人才特殊支持计划资助.
} 

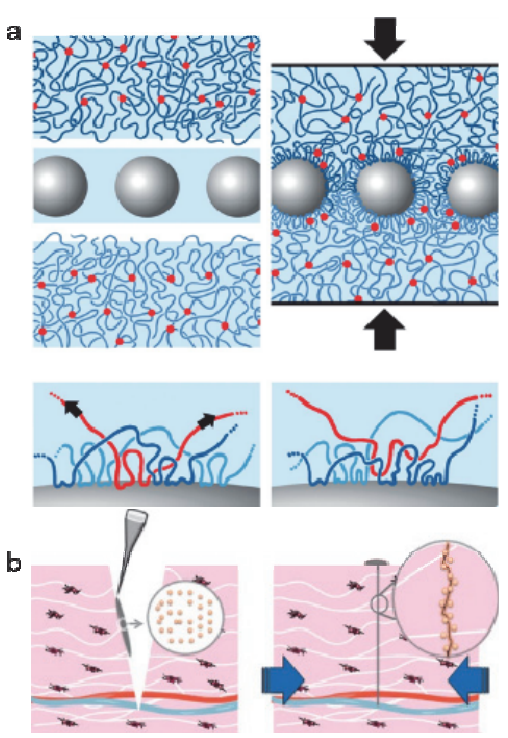

图 1 纳米颗粒溶液粘合剂. (a) 纳米颗粒溶液在凝胶之间的粘附示意 图 ${ }^{[7]}$; (b) 纳米颗粒溶液在伤口缝合中的应用 ${ }^{[8]}$.

Figure 1 Nanoparticle solutions adhesives. (a) The adhesion schematic illustration of nanoparticle solutions between two gel pieces ${ }^{[7]}$. (b) The application of nanoparticle solutions for wound closure ${ }^{[8]}$.

甚至低于 $0.08 \mathrm{MPa}$ ，两者的粘附差异比值最高可达 100 . 这种材料的各向异性粘附性质超过仿生微加工制备的 粘附材料和商业使用的胶水.
纤维素纳米晶体 $(\mathrm{CNCs})$ 实现定向自组装分为三个 阶段：(1)悬浮液的干燥边缘使 CNCs 局部浓缩并向接触 区域中心裉去; (2)当 CNCs 浓度达到临界阈值，主平面 内的内应力克服凝胶悬浮液的内聚力, 裂缝出现并开始 传播; (3) CNCs 片层通过超分子相互作用而凝固, CNCs 之间以及 CNCs 与基底之间毛细力的耦合作用使两个接 触面间形成高的内聚力和粘附力. 在超分子作用形成过 程中, 纤维素链在每个 CNCs 晶区内排列, 最终沿着薄 片层长轴方向排列, 实现 CNCs 定向自组装. 扫描电子 显微镜(SEM)、偏光显微镜以及原位小角 $\mathrm{X}$ 射线(SAXS) 等可以证明 CNCs 薄片层之间的断裂以及 $\mathrm{CNCs}$ 的自组 装定向排列行为.

纤维素纳米晶体悬浮液能够产生较强粘附是由两 个因素共同决定的: CNCs 通过超分子相互作用定向排 列形成高度有序的向列相微结构, 一方面 CNCs 与基底 形成超分子键和强毛细力相互作用, 赋予了高强度的界 面粘附; 另一方面 CNCs 本身具有很高的强度, 其抗拉 强度高于 $7 \mathrm{GPa}$, 轴向拉伸模量高于 $140 \mathrm{GPa}$, 因此, 在 平面内剪切时, 片层结构中沿纤维素链方向排列的 $\mathrm{CNCs}$ 产生的摩擦会导致较强的自粘附.

与传统复杂路线合成的粘合剂不同, Tardy 等 ${ }^{[9]}$ 仅利 用纤维素钠晶体和水, 通过简单的溶剂蒸发诱导纤维素 钠晶体自组装, 获得了具有超高各向异性粘附强度的胶

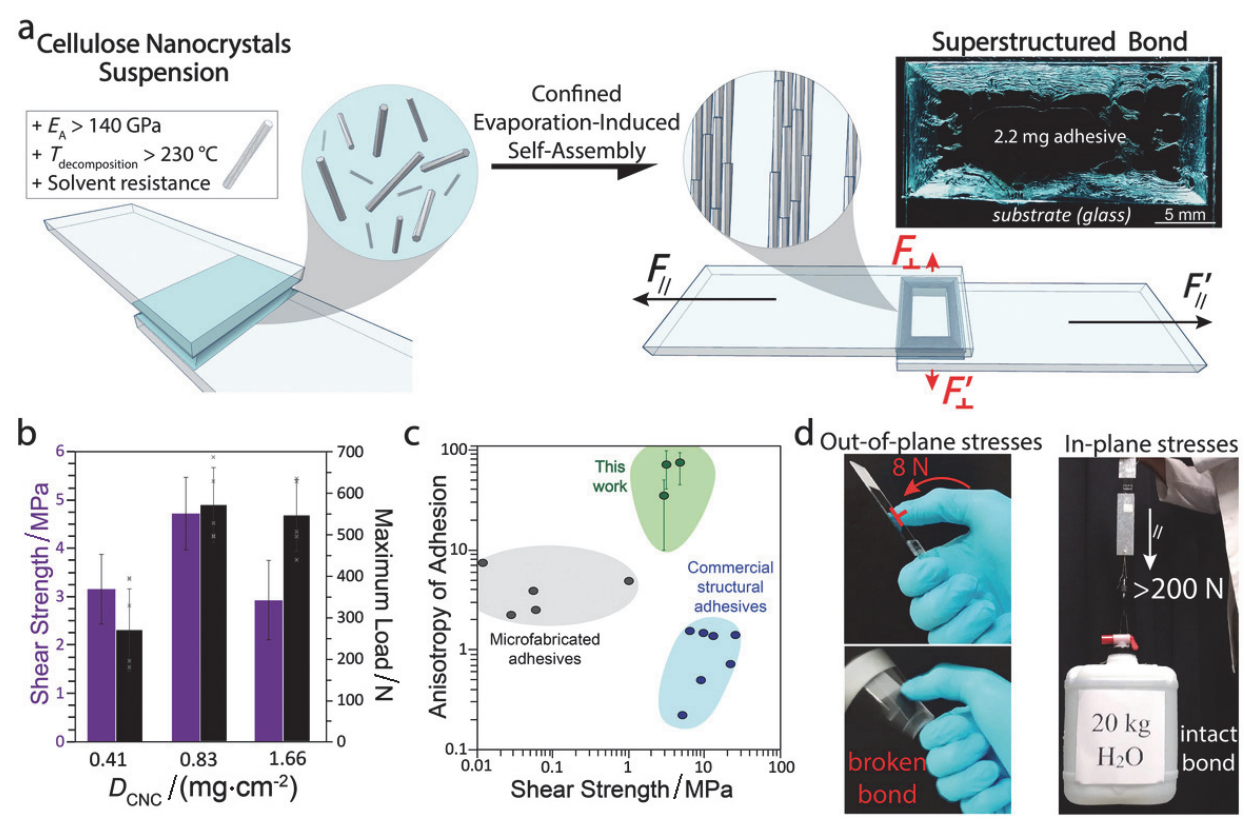

图 2 亲水性基材(如载玻片)之间的受限蒸发诱导自组装导致粘附和 CNCs (纤维素纳米晶体)超结构 ${ }^{[9]}$ : (a) CNCs 自组装示意图; (b) 在粘合剂与基 底接触区域内, 沉积三个不同 $\mathrm{CNC}$ 密度, 平面内的剪切粘附强度和对应承受的最大负载; (c) 根据面内与面外(垂直面内)粘附强度的比值(平均值 为 $73 \pm 25$ )计算得到本工作中的各向异性粘附差异远远高于微制造法和商用胶水; (d) 各向异性粘附示意图: 平面外的拇指法向按压去除粘附和平 面内的承重超过 $200 \mathrm{~N}$.

Figure 2 Confined evaporation-induced self-assembly between hydrophilic substrates (glass slides), resulting in adhesive and superstructured CNCs (cellulose nanocrystals) $^{[9]}$. (a) Schematic illustration of the self-assembly of CNCs. (b) Adhesive shear strength in-plane and corresponding maximum loads for three different areal densities of deposited CNC in the contact area between adhesives and substrate. (c) Anisotropy of adhesion in this work calculated from the ratio between the in-plane and out-of-plane (normal to in-plane), averaged $73 \pm 25$, is much larger than that of microfabrication approaches and commercial glues. (d) Illustration of the anisotropic adhesion: Thumb press normal to the plane (out-of-plane) removes the adhesive, and $>200 \mathrm{~N}$ in-plane load. 
水. 该粘合剂展现了优异的粘附性能，而且制备成本极 低, 为绿色环保粘合剂和可持续发展的新材料制备提供 了新的思路.

\section{作者简介}

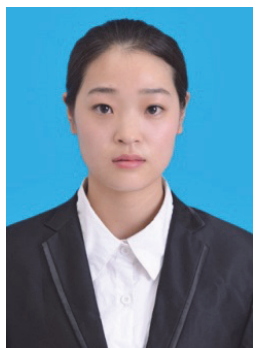

刘明倩, 2017 年毕业于兰州大学化学化工学院, 2017 年至 今为中国科学院理化技术研究所仿生材料与界面科学重点实 验室的直博生, 师从王树涛研究员, 主要研究方向为仿生湿 态粘附材料.

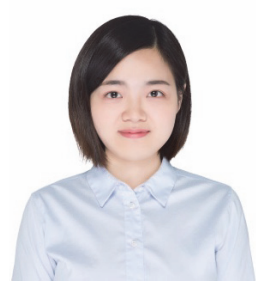

万茜子, 2015 年毕业于北京化工大学材料科学与工程学 院, 2015 年至今为中国科学院理化技术研究所仿生材料与界 面科学重点实验室的直博生, 师从王树涛研究员, 主要研究 方向为仿生湿态粘附界面材料.

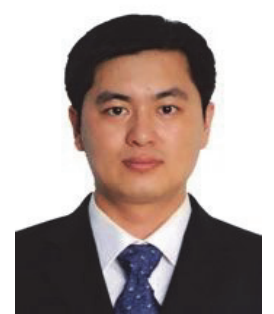

王树涛, 中国科学院理化技术研究所研究员. 2007 年博士 毕业于中国科学院化学研究所, 2007 年至 2010 年在美国加州 大学洛杉矶分校医学院从事博士后研究. 2010 年至 2014 年作 为研究员/课题组长就职于中国科学院化学研究所, 2014 年至 今就职于中国科学院理化技术研究所. 主要从事仿生粘附界 面材料与化学的研究, 如仿生粘附材料、抗粘附界面材料、生 物识别粘附界面和器件以及疾病早期诊断等方面的研究.

\section{References}

[1] Jeong, H. E.; Lee, J.-K.; Kim, H. N.; Moon, S. H.; Suh, K. Y. Proc. Natl. Acad. Sci. U. S. A. 2009, 106, 5639.

[2] Iturri, J.; Xue, L. J.; Kappl, M.; Garcia-Fernandez, L.; Barnes, W. J. P.; Butt, H. J.; Campo, A. D. Adv. Funct. Mater. 2015, 25, 1499.

[3] Baik, S.; Kim, D. W.; Park, Y.; Lee, T.-J.; Bhang, S. H.; Pang, C. Nature 2017, 546, 396.

[4] Jenkins, C. L.; Meredith, H. J.; Wilker, J. J. ACS Appl. Mater. Interfaces 2013, 5, 5091 .

[5] Zhao, Q.; Lee, D. W.; Ahn, B. K.; Seo, S.; Kaufman, Y.; Israelachvili, J. N.; Waite, J. H. Nat. Mater. 2016, 15, 407.

[6] Harris, T. I.; Gaztambide, D. A.; Day, B. A.; Brock, C. L.; Ruben, A L.; Jones, J. A.; Lewis, R. V. Biomacromolecules 2016, 17, 3761.

[7] Rose, S.; Prevoteau, A.; Elziere, P.; Hourdet, D.; Marcellan, A.; Leibler, L. Nature 2014, 505, 382.

[8] Meddahi-Pelle, A.; Legrand, A.; Marcellan, A.; Louedec, L.; Letourneur, D.; Leibler, L. Angew. Chem. Int. Ed. 2014, 53, 6369.

[9] Tardy, B. L.; Richardson, J. J.; Greca, L. G.; Guo, J. L.; Ejima, H.; Rojas, O. J. Adv. Mater. 2020, 32, e1906886.

(Cheng, B.) 